\title{
Case Report of the PET Scan Changes and Clinical Effects of Testosterone in the Treatment of a Stroke Patient
}

\author{
Robert S. Tan ${ }^{*, 1,4,5}$ and Shanker Raja ${ }^{2,3}$ \\ ${ }^{I}$ Geriatrics \& Extended Care, Michael E DeBakey VAMC, USA; ${ }^{2}$ Radiology \& Nuclear Medicine, Michael DeBakey \\ VAMC, USA; ${ }^{3}$ Baylor College of Medicine; ${ }^{4}$ University of Texas; ${ }^{5}$ Opal Medical Clinic, Houston, USA
}

\begin{abstract}
Testosterone has been known to improve functionality in stroke patients. In this case, we sought to illustrate the possible cognitive effects of testosterone replacement therapy with the aid of PET scanning. Glucose metabolism generally worsened in this patient after 12 weeks of treatment. However, there was improved glucose metabolism (CGM) in the occipital lobe, middle temporal gyrus and cerebellum. The middle temporal gyrus is the location of the hippocampus, which is part of the limbic system and plays important roles in long term memory and spatial navigation. The patient reported that he was sharper after testosterone, and clinical testing showed modest improvements in performance. This case finding needs to be confirmed with larger randomized controlled studies; but affords a window to the possibility of adding testosterone for the rehabilitation of stroke patients.
\end{abstract}

Keywords: Stroke, Testosterone, Hypogonadism, PET.

\section{INTRODUCTION}

It has been reported that testosterone administration in stroke patients may help in their rehabilitation [1]. We report the effects of a popularly prescribed topical testosterone (AndroGel ${ }^{\circledR} 1 \%$, Abbott Laboratories) in the treatment of a hypogonadal elderly patient with remote history of a left parietal cerebrovascular accident. The patient was transferred to us from another facility in New Orleans. When he arrived in Houston, he was noted to be aphasic but had no other focal neurological deficits. He was admitted to the transitional care unit for rehabilitation. He has a past medical history of diabetes, hypertension and coronary artery disease. Patient was found to be borderline hypogonadal as well and had a baseline total testosterone level of $307 \mathrm{ng} / \mathrm{dL}$ and free testosterone of $10.9 \mathrm{pg} / \mathrm{mL}$. Baseline prostatic-specific antigen (PSA) was $1.710 \mathrm{ng} / \mathrm{mL}$. He was clinically assessed to have primary hypogonadism secondary to age-related Leydig cell failure. Testosterone was initiated as part of a rehabilitative effort for his stroke.

\section{MATERIAL \& METHODS}

The patient underwent baseline clinical evaluation prior to having testosterone levels therapeutically elevated to a eugonadal state with AndroGel ${ }^{\circledR}$ (Abbott Laboratories) $5 \mathrm{~g}$ topical gel (containing $50 \mathrm{mg}$ testosterone) daily. Following informed consent, the patient underwent PET imaging: he was injected with ${ }^{18} \mathrm{~F}$ fluorodeoxyglucose (FDG) 15 minutes after an IV line had been placed and was placed in a quiet environment. The patient completed a computerized mental rotation task (based on the Shepard and Metzler paradigm) just prior to undergoing PET imaging; the purpose of this

* Address correspondence to this author at the Extended Care (110), Michael DeBakey VAMC, 2002 Holcombe Blvd., Houston, TX 77030, USA; Tel: (713) 791 1414; E-mail: robert.tan@va.gov activity is to stimulate the brain during the period of tracer uptake and standardize conditions of FDG uptake. Cerebral glucose metabolism was then measured by positron emission tomography (PET) (Philips Gemini GXL).

The patient underwent additional clinical testing and PET imaging at 12 weeks interval. The patient remained on TRT for a total of 6 months, and had a final round of clinical assessments done at that time. The clinical measures chosen for the evaluation are widely used, standardized functional measures including ADLs, IADLs and MMSE.

To compare and estimate the Z-score differences between age matched normal population database; both the baseline and post-therapy PET scans were analyzed on a PET-CT display workstation and neuroanalysis software (MIMvista corp, Cleveland OH, USA).

\section{RESULTS}

The patient had regular monitoring of testosterone, $\mathrm{H} \& \mathrm{H}$ and PSA, as shown in Table $\mathbf{1}$.

The stability of hemoglobin and PSA suggests safety at least in the short term. The baseline PET showed significant hypometabolism in the left parietal region of the brain, which was consistent with his history of stroke and persistent expressive speech deficits. Post treatment PET scan performed 12 weeks after the baseline assessment showed findings described below.

The baseline PET showed significant hypometabolism, particularly in the left hemisphere; this would be consistent with the history of stroke and persistent expressive speech deficits. The on-treatment PET scan showed similar persistent hypometabolism in the left hemisphere, without significant changes (see Figs. 1, 2). 


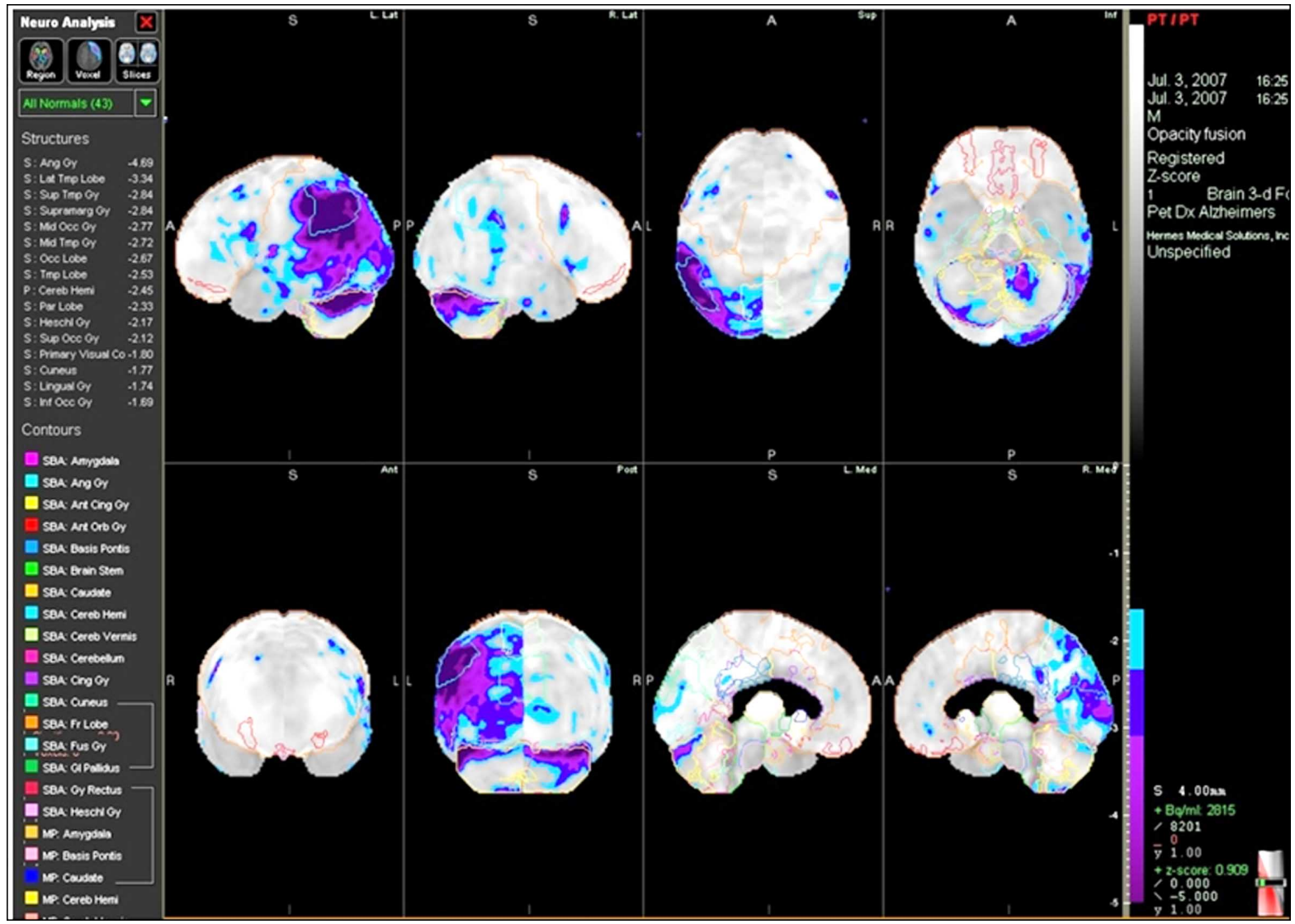

Fig. (1). Before TRT.

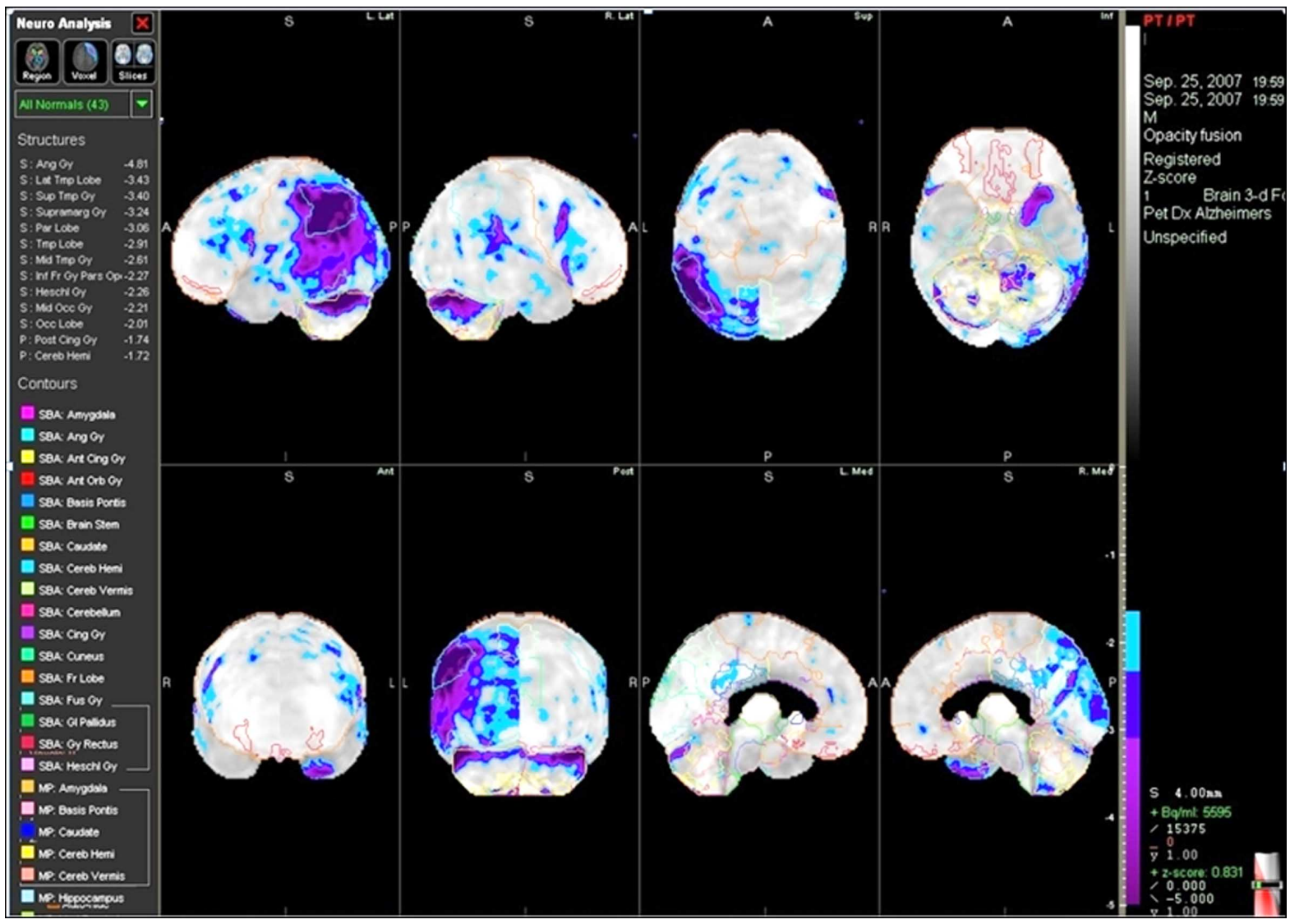

Fig. (2). After TRT. 
Table 1. Laboratory Values at Baseline and on Treatment

\begin{tabular}{|c|c|c|c|}
\hline & Baseline & On Treatment & Normal Range \\
\hline \hline Testosterone, total & $307 \mathrm{ng} / \mathrm{dL}$ & $457 \mathrm{ng} / \mathrm{dL}$ & $330-900 \mathrm{ng} / \mathrm{dL}$ \\
\hline Hemoglobin & $15.3 \mathrm{~g} / \mathrm{dL}$ & $15.9 \mathrm{~g} / \mathrm{dL}$ & $12.0-18 \mathrm{~g} / \mathrm{dL}$ \\
\hline PSA & $1.710 \mathrm{ng} / \mathrm{mL}$ & $1.820 \mathrm{ng} / \mathrm{mL}$ & $0-4 \mathrm{ng} / \mathrm{mL}$ \\
\hline
\end{tabular}

$\mathrm{Z}$ scores were compared pre and post treatment, and the differences noted. (Table 2) There was either improvement or worsening, with the most significant areas highlighted below:

1. Disease or stroke side (left) improvements:

a. Cerebellar hemispheres (baseline $\mathrm{z}=-1.66$ compared with follow up $\mathrm{z}=-0.62$, diff 1.04) It is to be noted that the right side also improved but to a lesser extent (with difference in $\mathrm{z}$ of 0.75 ).

b. Occipital lobe (baseline $\mathrm{z}=-4.53$ compared with follow up $\mathrm{z}=-3.53$, diff 1.0 ).

c. In particular, the middle occipital gyrus improved by a difference of 1.5. However, it is to be noted that there was improvement in the occipital lobe of albeit less than 0.51 on the contralateral non-disease side.

d. Middle temporal gyrus (baseline $\mathrm{z}=-4.87$ compared with follow up $\mathrm{z}=-4.06$, diff 0.81$)$. The right side also improved, but to a lesser extent (0.13).

2. Disease or stroke side (left) worsening:

Notably, the amygdala in the left hemisphere had worsening (baseline $\mathrm{z}=4.0$ compared with follow up $\mathrm{z}=$ 1.03 , diff -2.99 . We note that the $\mathrm{z}$ was very positive at baseline and it appears to be returning back to a more normal z score.

3. Normal (right) improvements.

a. Anterior orbital gyrus had the greatest gain of 0.99 , from a baseline $\mathrm{z}$ score (1.36 to 2.35).

b. Fusiform gyrus also improved by 0.89 , from a baseline of -1.05 to -0.16 .

4. Normal (right) worsening.

The insula worsened by -2.46 , with a baseline of 2.38 and post treatment of -0.08 .

5. In general, most areas had less glucose metabolism after testosterone therapy. In particular, the frontal lobe, insular, cingular gyrus, amygdalae were the areas that demonstrated decreased glucose metabolism after testosterone therapy. For the frontal lobe, insular and cingular gyrus, the worsening of glucose metabolism was more pronounced on the normal side or right side. The amygdalae got significantly worse glucose metabolism on the left side.

The patient's clinical test scores after 6 months on testosterone therapy were compared with his baseline performance. Subjectively, he did report that he was "brighter and sharper"; and his MMSE improved from 15 out of 30 to 19 out of 30 .

\section{DISCUSSION}

In epidemiological studies of older men, low testosterone and conversely high estradiol levels have been associated with a higher risk for cerebrovascular events [2, 3]. We are not clear if the patient was hypogonadal before the stroke or as a result of the stroke; and thus cannot infer that his hypogonadal state put him at risk for the stroke. Our aim was to study the effects of testosterone in the brain both functionally as well as visually by imaging. We are making the assumption that PET scanning gives an insight of how the brain functions, and acknowledge the limitation of this tool as the brain is more complex. However, glucose metabolism does give us an idea of how metabolically active particular parts of the brain are at a particular time.

Modest improvements in clinical functioning were noted in the patient and it correlated with the PET scan findings. It is to be noted that testosterone appeared to improve some areas and seemed also to worsen some other areas. While we may not fully understand this, we do hypothesize that glucose metabolism was redirected to other areas. This may also change with time, in that the brain being so plastic, may regulate glucose metabolism in different parts of the brain at different times.

The patient did improve in balance and visual spatial skills after clinical assessment. This tied in well with the improvements in glucose uptake in the cerebellum and occipital lobe on the left or diseased side of the brain. The gain in anterior orbital gyrus on the normal side may have also contributed to the improved visual spatial status of this patient. There was improvement in the medial temporal lobe, which is the seat of the hippocampus. The hippocampus is a major component of the brains of humans. The hippocampus belongs to the limbic system and plays important roles in long term memory and spatial navigation. A single case report cannot infer causality, but further research is needed to establish if this observation can be repeated in other studies.

The areas in the brain that got worse after testosterone treatment may need some explanation. Notably, the amygdala got worse on the diseased side. The amygdala is the seat of memory and emotions and a previous study suggested improvements in memory [4] with testosterone replacement, which may be contrary to what we have found here. It may be that testosterone effects in the brain may change with time, and that this area will improve at a different stage and this has to be explored in another study. On the normal side, we found that the insula got worse glucose metabolism as compared to baseline. The insula is the seat for emotion and body homeostasis. We cannot conclude that testosterone does not help with improving 
Table 2. Selected areas of Brain with Increase or Decrease in CGM

\begin{tabular}{|c|c|c|c|c|}
\hline & Baseline Left (Stroke) & Baseline Right (No Stroke) & Change Left (Stroke) & Change Right (No Stroke) \\
\hline \multicolumn{5}{|l|}{ Cerebellum } \\
\hline Cerebellar Hemisphere & -1.66 & -1.42 & 1.04 & 0.75 \\
\hline \multicolumn{5}{|l|}{ Frontal } \\
\hline Anterior orbital gyrus & 0.78 & 1.36 & -0.11 & 0.99 \\
\hline \multicolumn{5}{|l|}{ Interlobal } \\
\hline Insula & 1.20 & 2.38 & 0.06 & -2.46 \\
\hline \multicolumn{5}{|l|}{ Limbic } \\
\hline Nucleus Accumbens & -0.10 & 2.59 & 0.82 & -0.34 \\
\hline \multicolumn{5}{|l|}{ Occipital } \\
\hline Occipital Lobe & -4.53 & -1.24 & 1.00 & 0.51 \\
\hline Inferior Occipital Gyrus & -3.29 & 0.02 & 1.06 & 0.36 \\
\hline Middle Occipital Gyrus & -5.98 & -0.69 & 1.50 & 0.66 \\
\hline Superior Occipital Gyrus & -3.37 & -1.24 & 0.86 & 0.82 \\
\hline \multicolumn{5}{|l|}{ Parietal } \\
\hline Parietal Lobe & -4.82 & 0.99 & -0.06 & -1.05 \\
\hline Inferior Temporal Gyrus & -2.65 & -0.34 & 0.66 & 0.87 \\
\hline Middle Temporal Gyrus & -4.87 & -0.77 & 0.81 & 0.13 \\
\hline Temporal Pole & 1.50 & 2.56 & -1.78 & 0.16 \\
\hline \multicolumn{5}{|l|}{ Other } \\
\hline Globus Pallidus & 2.38 & 4.36 & 0.41 & -1.37 \\
\hline Putamen & 1.47 & 2.63 & -0.25 & -1.34 \\
\hline Thalamus & 1.21 & 3.43 & -0.29 & -1.44 \\
\hline
\end{tabular}

emotions or body homeostasis, but only can admit that the brain is very complex and that emotions and body homeostasis is controlled by a variety of areas of the brain.

Thus, treatment with a topical testosterone (AndroGel ${ }^{\circledR}$ $1 \%$ ) demonstrated improved cerebral glucose metabolism in some parts of the brain, but some areas paradoxically got worse.

A group study with a repeated measure design will be necessary to determine if clinical findings from the current study are clearly attributable to treatment effects or to other non-specific factors (e.g. practice effects). Changes in neurometabolism noted on PET scanning, however, provide some evidence of direct neurological effect of testosterone replacement. It is well known that testosterone can influence visual spatial domains in normal patients [5] and it is possible that modest improvements in visual skills of the current patient were attributable to testosterone replacement. This may have important implications for the rehabilitation of brain injured patients with visual deficits. Testosterone 
may also have beneficial effects on muscle strength [6] and hence mobility in brain injured populations, although these domains were not assessed in the current study. Future studies will be necessary to clarify the mechanism of action of testosterone in brain injured individuals and also to determine if our current PET scan techniques are sensitive enough to measure metabolic changes that are induced by testosterone.

\section{CONFLICT OF INTEREST}

For this case study, neither the patient nor the investigator received any payment. The assessment tools, medication and PET scans were funded through a grant from Abbot Laboratories.

\section{ACKNOWLEDGEMENTS}

This work was supported, in part, by an investigatorinitiated grant from Abbott Laboratories. Abbott had no role in the project design, in data collection, analyses, or interpretation of the results. We thank Javier Villanueva-
Meyer MD, Diego I Florentin MD and Liudmila Buell MD for their invaluable help in various aspects of this project.

\section{REFERENCES}

[1] Okamoto S, Sonoda S, Tanino G et al., Change in thigh muscle cross-sectional area through administration of an anabolic steroid during routine stroke rehabilitation in hemiplegic patients. Am J Phys Med Rehabil 2011;90(2): 106-11.

[2] Morales A. Words of wisdom. Re: lower testosterone levels predict incident stroke and transient ischemic attack in older men. Eur Urol 2010; 58(1): 182-3.

[3] Yeap BB, Hyde Z, Almeida OP, et al., Lower testosterone levels predict incident stroke and transient ischemic attack in older men. $\mathbf{J}$ Clin Endocrinol Metab 2009; 94: 2353-9.

[4] Tan and Pu SJ. A pilot study on the effects of testosterone in hypogonadal aging male patients with Alzheimer's disease. Aging Male 2003; 6(1): 13-7.

[5] Holland J, Bandelow S, Hogervorst E. Testosterone levels and cognition in elderly men: a review. Maturitas 2011; 69(4): 322-37.

[6] Bakhshi V, Elliott M, Gentili A et al., Testosterone improves rehabilitation outcomes in ill older men. J Am Geriatr Soc 2000; 48(5): 550-3. 